\title{
THE PRECISION OF GOOGLE EARTH MAP ANALYSIS WITH THE COORDINATES OF IGS STATIONS
}

\author{
Xuwang Wang ${ }^{1}$, Fan Wang ${ }^{2}$ \\ 1Dalian University of Technology, Dalian city, Liaoning provice, China - wxwang007@163.com \\ ${ }^{2}$ National Geomatics Center of China, Beijing, China - wangfan@ngcc.cn
}

KEY WORDS: Google Earth; IGS station; Coordinates; label; Identification; accuracy evaluation

\begin{abstract}
:
This paper identifies the location of global IGS reference station on Google Earth map, and raises a method of identifying the location and analysis the precise position accuracy. The Google Earth map supports WGS-84 geodetic coordinates. Acquire the WGS-84 Precise Geodetic Coordinates of the Global IGS reference station on IGS Website. The IGS reference station is labeled on the Google Earth map exactly in KML markup language. Using Google Earth ruler tool, calculated the map distance, ground distance and azimuth angle of the Google Earth map position and the labeled position from the precision IGS reference station. Identifying the deviations between the exactly point location of IGS station and the recognized location of Google Earth. About 1,054 IGS stations which covering all over the world are using as samples for data statistics. With a series of statics graph, analysis the accuracy and recognition rate. It shows that the recognition rate is $71.8 \%$ and the average accuracy is about 4.38 meters. The accuracy from $2 \mathrm{~m}$ to $4 \mathrm{~m}$ occupied $18.76 \%$; the accuracy from $4 \mathrm{~m}$ to $6 \mathrm{~m}$ occupied $41.0 \%$; the accuracy from $6 \mathrm{~m}$ to $8 \mathrm{~m}$ occupied $6.34 \%$; the accuracy from $8 \mathrm{~m}$ to $10 \mathrm{~m}$ occupied $3.70 \%$ etc. The accuracy in China region is about $1.5 \sim 2$ meters and it's reach up to 1 meter in the cities of the United States, Europe and Japan etc. So that Google Earth map could be used for high-precision positioning services, such as, field reconnaissance, survey network design and the description of station etc.
\end{abstract}

\section{INTRODUCTION}

The International GNSS Service (IGS) has about 1054 GNSS continuously operating reference stations (hereinafter referred to as IGS stations) all over the world, providing a series of GNSS information service globally by free of charge. The IGS stations continue observe and track satellite navigation signals in long term. Transfer the observation data to a fixed ground observation stations of the data center in real-time or a regular time by communication facilities. The GNSS antenna is placed at the top of the observation pier (Figure 1).

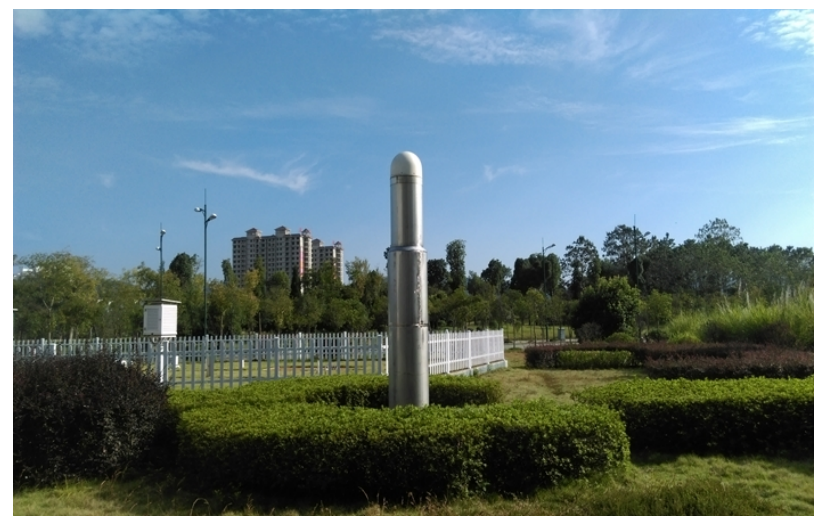

Figure 1. Continuously operating reference station

Google Earth (GE) is a Virtual Earth software developed by Google Company. It lays satellite photos, aerial photographs and GIS on a three-dimensional model of the Earth. The effective resolution of global landform images on Google Earth We developed a positioning program in KML markup language. is less than 100 meters, generally is 30 meters (for example, in the area of Chinese mainland), and the Eye ALT is about 15 kilometers (that is, the object with a width of 30 meters is only a pixel of the image, and it would be the mosaic when kept zoom in). For the big cities, famous scenic spots and the buildings area etc., a high-precision image with the resolution about $1 \mathrm{~m}$ or $0.5 \mathrm{~m}$ and the Eye ALT is about 500 meter and 350 meter will be provided. The most of the cities which have the highprecision images locate in North America and Europe. In other regions, only the national's capital and very important city has the high-precision images. Lots of area in China have their high-precision images, which including all most of big cities in Chinese mainland. In addition, the dams, oil fields, bridges, highways, harbors, wharfs and military airports are the key points of Google Earth.

This paper obtains the exact WGS-84 coordinate of IGS stations, converts all the coordinated into KML format, and marks it on Google Earth map. By comparing the marked precise coordinate points with the displayed position of Google Earth imaging, measuring the distance and azimuth angle between them. Identify and analysis the accuracy of IGS stations on the Google Earth map. Define the different level of the accuracy which could be provided by Google Earth map in different regions, and the suitable position service on it for the customers.

\section{POSITIONING THE GLOBLAL IGS STATIONS WITH PECISION COORDINATES}

Google Earth map supports WGS-84 geodetic coordinates. We download the precise WGS-84 coordinates of the 1054 reference stations from IGS website. The precise coordinates of IGS stations are programmatically marked on Google Earth map. All 1054 IGS station points are marked on Google Earth map 
with coordinate labeling. Below is the source codes:

$<\mathrm{kml}>$

$<$ Document $>$

$<$ name $>$ IGS Sites.kml $<$ name $>$

$<$ Folder $>$

$<$ name $>$ IGS Sites $</$ name $>$

$<$ open $>1<$ open $>$

$<$ Placemark $>$

$<$ name $>$ BUDP_GPS $</$ name $>$

$<$ description $>$ BUDP GPS $</$ description $>$

$<$ styleUrl $>\#$ GPSSiteStyle $</$ styleUrl $>$

$<$ Point $>$

$<$ coordinates $>12.50002552, \quad 55.73902020$,

$94.02690838</$ coordinates $>$

$<$ Point $>$

$</$ Placemark $>$

$<$ Placemark $>$

$<$ name $>$ BUEN 2PS $<$ /name $>$

$<$ description $>$ BUEN 2 PS $</$ description $>$

$<$ styleUrl $>$ \#GPSSiteStyle $<$ /styleUrl $>$

$<$ Point $>$

$<$ coordinates $>-77.01042040, \quad 3.88202318$,

$57.75344228</$ coordinates $>$

$<$ Point $>$

$<$ Placemark $>$

$</$ Folder $>$

$</$ Document $>$

$</ \mathrm{kml}>$

\section{IDENTIFY IGS STATION POINTS ON GOOGLE}

\section{EARTH MAP}

Showing the coordinates of the global IGS station on the Google Earth map. According to the precise coordinates of the IGS station points, identify the imaging position of the IGS station on the Google Earth map. Using Google Earth ruler tool, putting the start of ruler on the labeled point of the precision coordinate IGS station and the end of ruler on the center of the antenna image of IGS station on Google earth map, measure the map distance, ground distance and azimuth angle from the marked point with precision coordinate label to the imaging position of Google Earth map. Calculate the deviations between the exactly point location of IGS station and the recognized location by Google Earth.

\section{COMPARE AND ANALYSIS THE RECOGNIZED POSITION WITH THE MARKED PRECISE CORRDNATE POSITION}

\subsection{Recognition rate statistics}

1054 samples were measured totally. The 297 samples of them cannot be identified by person on the Google Earth map. In another word, the position of GNSS antenna can be found on the map view; and the location the 757 samples were identified. The recognition rate is $71.8 \%$.

The unrecognizable reasons are as follows: (1) The IGS station is on the top of mountain, snowfield, desert etc. The antenna cannot be found by person. (2) The resolution of image around the IGS station is too low to recognize clearly. (3) Many similar objects around the position of IGS station look like the GNSS antenna. It confuses the position of the exact antenna. (4) The image color of the marked coordinate point is very closely with the color of IGS station, it's hard to distinguish.

The situation of easy to identify: (1) The coordinate point locates in the big city, the famous scenic spots. (2) The coordinate point is in the area with a high resolution image of Google Earth map resolution. (3) The color of the coordinate point image is different with the photo of IGS station obviously.

\subsection{Analysis on Recognition Accuracy}

All 757 identified IGS stations are using as the samples for the data statistics and accuracy analysis. In this paper, we did data statistics and accuracy analysis on both distance and observing Pier high.

4.2.1 Accuracy Analysis in Distance: Amount the 757 recognized samples, the average distance between the marked positions of the precise coordinate IGS stations and the antenna position of IGS stations which recognized manual on the Google Earth map is 4.38 meters. The maximum distance is 57 meters. The accuracy of $0-2 \mathrm{~m}$ is occupied $41.08 \%$; the accuracy of $2 \mathrm{~m}-4 \mathrm{~m}$ is occupied $18.76 \%$; the accuracy of $4 \mathrm{~m}-6 \mathrm{~m}$ is occupied $20.61 \%$; the accuracy is over $6 \mathrm{~m}$ occupied $9.55 \%$. The deviation in distance statistics show in Table 1.

\begin{tabular}{|c|c|c|c|c|c|c|c|c|c|c|}
\hline $\begin{array}{l}\text { dist } \\
\text { ance } \\
\text { (cm) }\end{array}$ & $\begin{array}{l}{[0,1} \\
00]\end{array}$ & $\begin{array}{l}{[100,} \\
200]\end{array}$ & $\begin{array}{l}{[300,} \\
400]\end{array}$ & $\begin{array}{l}{[400,} \\
500]\end{array}$ & $\begin{array}{l}{[500,} \\
600]\end{array}$ & $\begin{array}{l}{[600,} \\
700]\end{array}$ & $\begin{array}{l}{[700,} \\
800]\end{array}$ & $\begin{array}{l}{[800,} \\
900\end{array}$ & $\begin{array}{l}{[900,} \\
1000 \\
\}\end{array}$ & $\begin{array}{l}\| 10 \\
00\end{array}$ \\
\hline $\begin{array}{l}\text { Nu } \\
\text { mbe } \\
\text { r }\end{array}$ & 156 & 142 & 114 & 87 & 67 & 43 & 30 & 18 & 17 & 89 \\
\hline $\begin{array}{l}\text { Perc } \\
\text { enta } \\
\text { ge }\end{array}$ & $\begin{array}{l}20.6 \\
1 \%\end{array}$ & $\begin{array}{l}18.7 \\
6 \%\end{array}$ & $\begin{array}{l}15.0 \\
6 \%\end{array}$ & $\begin{array}{l}11.4 \\
9 \%\end{array}$ & $\begin{array}{l}8.85 \\
\%\end{array}$ & $\begin{array}{l}\mathbf{5 . 6 8} \\
\%\end{array}$ & $\begin{array}{l}3.96 \\
\%\end{array}$ & $\begin{array}{l}2.38 \\
\%\end{array}$ & $\begin{array}{l}2.25 \\
\%\end{array}$ & $\begin{array}{l}11.4 \\
5 \%\end{array}$ \\
\hline
\end{tabular}

Table 1. Deviation in distance statistics

Distance deviation distribution statistics and analysis: 757 sample points Accuracy Distribution (Figure 2 6).

Figure 2 is a pie of accuracy distribution. The accuracy of 156 stations is less than $1 \mathrm{~m}$; The accuracy of 142 stations is in $1 \mathrm{~m}$ $\sim 2 \mathrm{~m}$; The accuracy of 114 stations is in $3 \mathrm{~m} \sim 4 \mathrm{~m}$ etc.

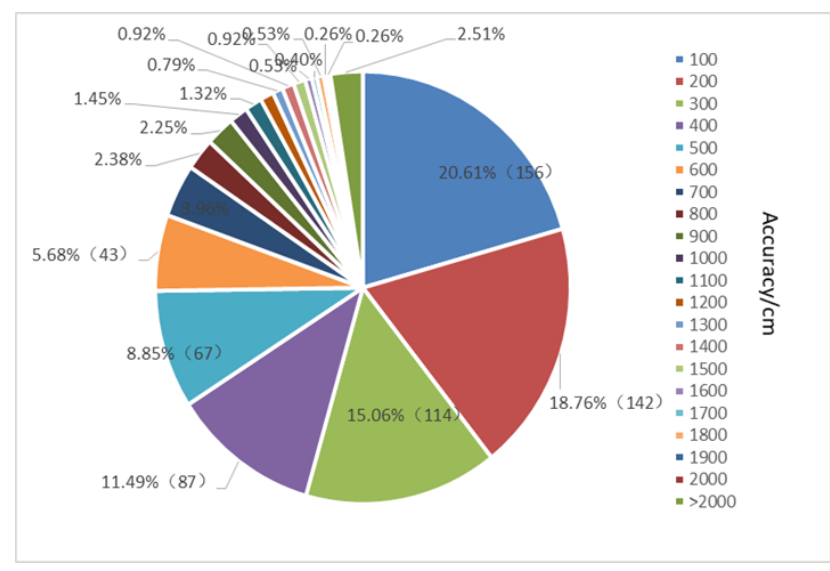

Figure 2. Accuracy distribution

It shows that 156 points have the accuracy of $0-1 \mathrm{~m}$, it is $20.61 \%$ of all recognized points; the accuracy within 5 meters is about $63.28 \%$; the accuracy within 10 meters is higher to $90 \%$. Only 10 points have the accuracy lower to 25 meters. As the precision distance increases, the resolution decreases and the number of points decreases. 
4.2.2 Accuracy Analysis in Distance component: calculate the deviations in different distance component of the all 757 samples. Basing to the map distance $\mathrm{L}$ and azimuth angle $\theta$, calculate the deviation on the direction of east-west Ly and the deviation on the direction of north-south Lx separately.

$$
\mathrm{Ly}=\mathrm{L} * \sin \theta ; \quad \mathrm{Lx}=\mathrm{L} * \cos \theta
$$

Where $\quad \mathrm{L}=$ map distance

$\Theta=$ azimuth angle

Ly $=$ deviation on East-West

$\mathrm{Lx}=$ deviation on North-South

Figure 3 shows the accuracy of most points is less than 10 meters in map distance.

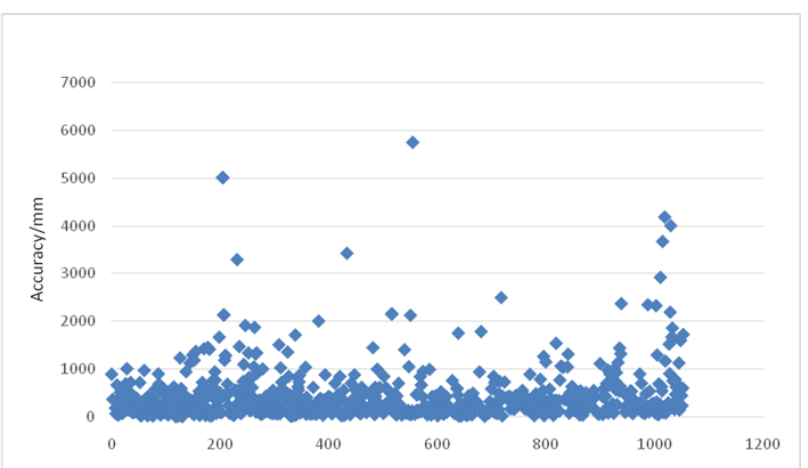

Figure 3. Accuracy (map distance) of IGS stations

The North-South component of each point is represented by "N". "+" stands for the deviations in North and "-" stands for the South direction; The East-West component is represented by "E". "+" stands for the deviations in North and "-." stands for the south direction. The vertical component (altitude difference) is always "U".

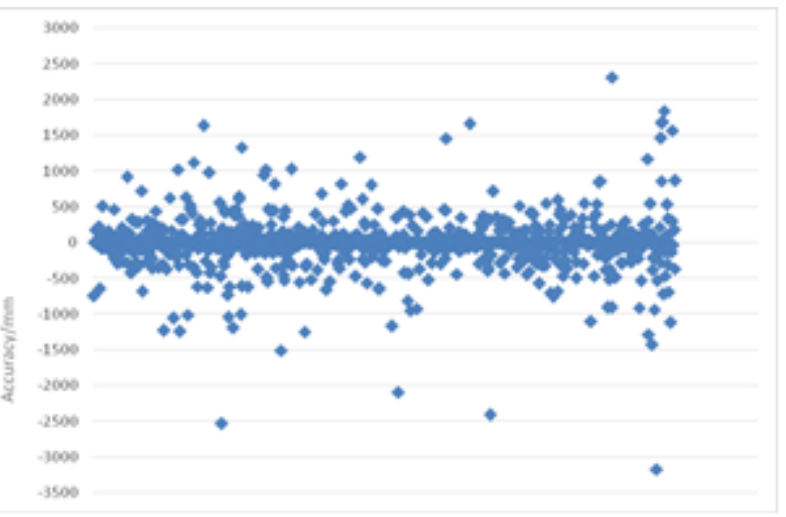

Figure 4. Accuracy (map distance) in North-South direction

The statistical graph shows that the $\mathrm{N}$ accuracy on most points is about 5 meters (in Figure 4). And the E accuracy is about 5 meters on all most of points (in Figure 5).

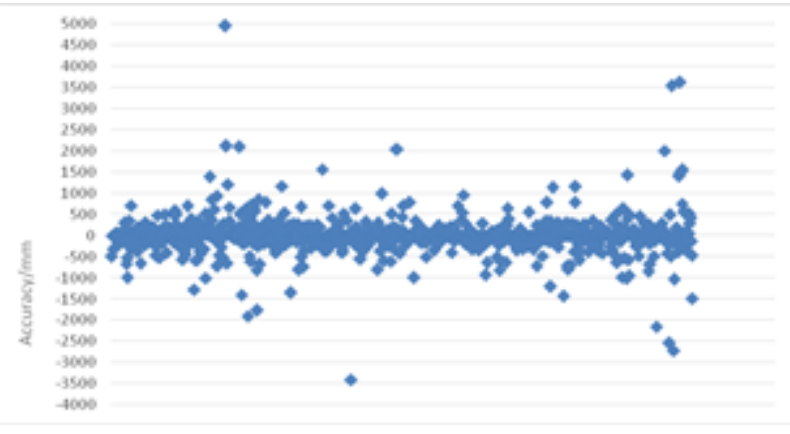

Figure 5. Accuracy (map distance) in East-West direction

4.2.3 Accuracy Analysis on the height of Observation Pier: Statistics and analysis the deviation of vertical component. The altitude difference $\mathrm{H}$ is calculated by the map distance $\mathrm{L}$ and ground distance $\mathrm{D}$ with the below equation

$$
H=\sqrt{D^{2}-L^{2}}
$$

Where: $\quad \mathrm{L}=$ map distance

$$
\begin{aligned}
& \mathrm{D}=\text { ground distance } \\
& \mathrm{H}=\text { altitude difference }
\end{aligned}
$$

According to the accuracy of altitude difference statistics, In 757 sample points (Figure 6), the altitude difference of 463 sample points is 0 . That is to say, $44 \%$ of samples cannot be identified by the observation pier height of IGS station. But $56 \%$ of samples can be identified by the observation pier height. Please find the details in below statistical graph.

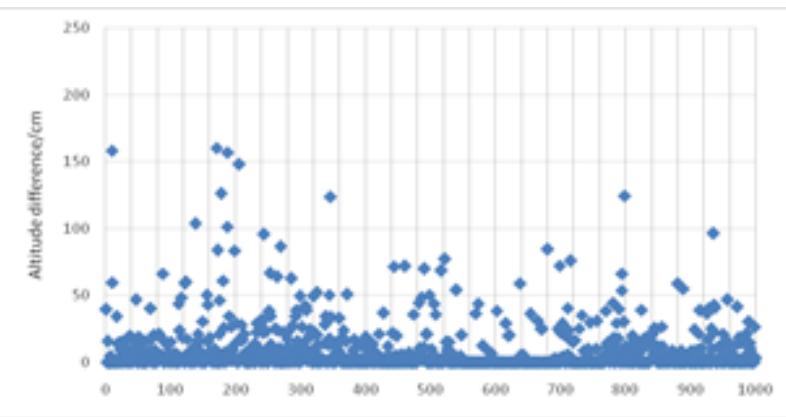

Figure 6. Altitude difference distribution map

The maximum altitude difference of the observation pier is 2.14 meters. The height of observation piers at most points can be identified. And most of observation piers height is around 1 meter.

\section{RECONGNITION ACCURACY OF THE GLOBAL IGS STATION DISTRIBUTE}

All the recognizable 757 IGS stations were pinned on the world map with their recognition accuracy. According to the accuracy distribution map in Figure 8, we can see, the IGS stations in the United States, Europe and Japan have a high recognition accuracy generally. The high accuracy could be 1 meter. In other regionslcountries, the recognition accuracy usually is lower. For the points of island area, the accuracy is not good too. 


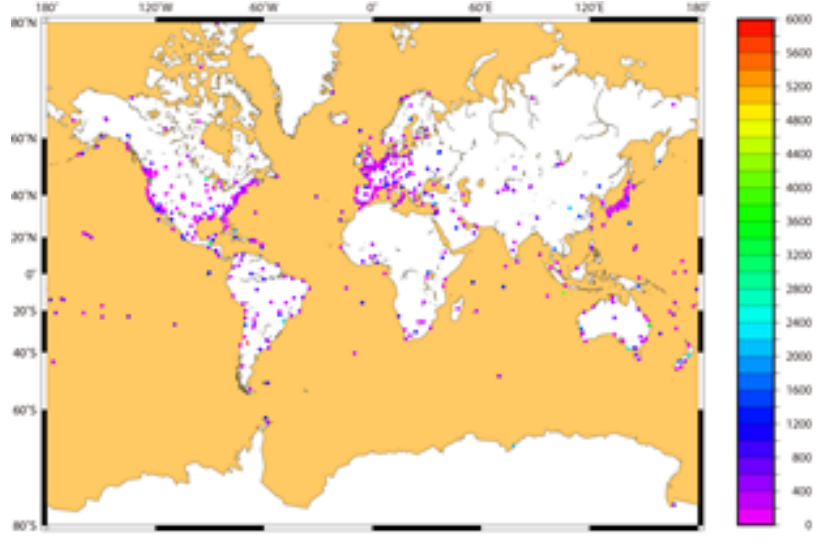

Figure 7. IGS stations - accuracy distribution map

\section{CONCLUSION}

In this paper, we chose about 1,054 IGS stations with precision coordinates all over the world as the samples to do data statics and analysis. Compare the marked the precision coordinates points of IGS station with the recognized position of the GNSS antenna on IGS station in Google map. Base on the value of the recognition accuracy and the identified rate, it shows that 297 samples can't be identified their location on the Google Earth map, 757 samples can be identified. The recognition rate is about $71.8 \%$. The average accuracy is about 4.38 meters. The accuracy ranging from $2 \mathrm{~m}$ to $4 \mathrm{~m}$ occupied $18.76 \%$ of total points; ranging from $4 \mathrm{~m}$ to $6 \mathrm{~m}$ occupied $41.0 \%$, ranging from $6 \mathrm{~m}$ to $8 \mathrm{~m}$ occupied $6.34 \%$, ranging from $8 \mathrm{~m}$ to $10 \mathrm{~m}$ occupied $3.70 \%$, ranging from $10 \mathrm{~m}$ to $12 \mathrm{~m}$ occupied $2.38 \%$, ranging from $12 \mathrm{~m}$ to $14 \mathrm{~m}$ occupied $1.72 \%$, ranging from $14 \mathrm{~m}$ to $16 \mathrm{~m}$ occupied $1.45 \%$. The accuracy in China region is about 1.5 2 meters and it's up to 1 meter in the cities of the United States, Europe and Japan etc.

So that Google Earth can be used for high-precision positioning services, such as, field reconnaissance, survey network design and the description of station etc.

\section{REFERENCES}

Hongquan Yang, Application Status and Development Trend of Mass Electronic Map [J].Surveying and Mapping Bulletin, 2014 (11) : 36-41

Dingliang Yang, Zhiping liu, Chongwu Zhong, Accuracy analysis of coordinate decryption method for Baidu Map [J]. Surveying and Mapping Bulletin, 2018 (7) 43-47

Weiping Jiang, Data Processing Method and Application of GNSS Base Station Network [D]. Wuhan University Press. 2017

Yating Xue, Research on Design and Implementation Method of Digital Campus Based on Google Earth and KML [D]. Lanzhou University. 2007

Jie Zhang, Brief discussion on three-dimensional modeling method [J].Journal of Yuncheng University, 2005, 23 (2), 37-38

Fengjuan Wu. Dynamic Establishment of Geodetic Data Model [J]. Marine Surveying and Mapping, 2006, (4), 39-41

Challstrom C W. Input Formats and Specifications of the National Geodetic Survey Data Base Volume I. h Horizontal Control Data[S]. The NGS Bluebook, 1998. 12 18 